\title{
An Argument-Principle Based Stability Criterion and Application to the Design of IIR Digital Filters
}

\author{
Wu-Sheng Lu \\ Dept. of Electrical and Computer Engineering \\ University of Victoria \\ Victoria, BC, Canada V8W 3P6 \\ Email: wslu@ece.uvic.ca
}

\begin{abstract}
A method for the design of IIR digital filters with pole radius constraint based on the argument principle (AP) from complex analysis is proposed. Unlike the stability constraints available in the literature which are sufficient but not necessary stability constraints, the proposed AP-based stability condition is both sufficient and necessary. We show that weighted leastsquares and minimax designs of robustly stable IIR filters can be accomplished in convenient convex programming settings where the proposed stability criterion can easily be incorporated as a single equality constraint that does not depend on any parameters other then filter's denominator coefficients. Design examples are presented to illustrate the usefulness of the proposed algorithms.
\end{abstract}

\section{INTRODUCTION}

A critical problem encountered in the design of IIR filters is stability. The stability problem cannot be adequately addressed by simple stabilization techniques such as replacing the poles of the transfer function outside the unit circle with their reciprocals if the passband phase response of the filter is a part of the design considerations. A recent research trend is to deal with the design problem in a constrained optimization setting, see for example [1]-[7] and the references cited therein. Among other things, a successful recent attempt to use complex-analysis tools to tackle the stability problem is described in [3] where Rouché's theorem [8] is applied to derive a family of linear constraints that ensures filter's stability. The method of [3] has however two drawbacks: the linear constraints depends on a parameter $\theta$ which varies continuously on $[0, \pi]$ that leads to a complicated semi-infinite optimization problem and, in addition, Rouché's theorem is merely a sufficient (but not a necessary) condition for the stability, the use of which may therefore exclude good designs. A method based on positive realness (PR) which offers improved stability region is proposed in [5]. Again the PR-based stability condition is a sufficient condition.

In this paper, we derive a stability criterion based on the argument principle from complex analysis [8]. The stability criterion obtained is a single equality constraint that does not depend on any parameters other than the denominator coefficients. Weighted least-squares and minimax designs of IIR filters are formulated as iterative convex quadratic and secondorder cone programming problems [9], respectively, where the proposed stability criterion can readily be incorporated to ensure stability. Design examples are presented to illustrate the proposed algorithms.

\section{A New Stability CRITERion}

Consider monic polynomial

$$
b(z)=\sum_{i=0}^{m} b_{i} z^{m-i}, \quad b_{0}=1
$$

Polynomial $b(z)$ is said to be $r$-Schur if all the zeros of $b(z)$ lie strictly inside circle $\mathcal{C}=\{z:|z|=r\}$ where $r \leq 1$. It is well known [8] that $b(z)$ is $r$-Schur if and only if

$$
\frac{1}{2 \pi j} \int_{\mathcal{C}} \frac{b^{\prime}(z)}{b(z)} d z=m
$$

where the integral is carried out counterclockwise along $\mathcal{C}$. Note that

$$
\frac{1}{2 \pi j} \int_{\mathcal{C}} \frac{b^{\prime}(z)}{b(z)} d z=\frac{1}{2 \pi j} \int_{\mathcal{C}} d \ln b(z)=\frac{1}{2 \pi} \int_{\mathcal{C}} d[\arg b(z)]
$$

Therefore, $b(z)$ is $r$-Schur if and only if the total change in $\operatorname{argument} \arg b(z)$ is equal to $2 \pi m$ when $z$ moves along $\mathcal{C}$ counterclockwise. The above is essentially a statement of the argument principle (AP) [8]. In what follows, the AP is applied to derive a stability criterion suitable for the analysis and design of IIR digital filters with robust stability.

If we define $c_{r m}(\theta)=r^{m} \cos m \theta, s_{r m}(\theta)=r^{m} \sin m \theta$,

$$
\begin{aligned}
\boldsymbol{b} & =\left[\begin{array}{c}
b_{1} \\
\vdots \\
b_{m}
\end{array}\right], \boldsymbol{c}_{r}(\theta)=\left[\begin{array}{c}
r^{m-1} \cos (m-1) \theta \\
\vdots \\
r \cos \theta \\
1
\end{array}\right], \\
\boldsymbol{s}_{r}(\theta) & =\left[\begin{array}{c}
r^{m-1} \sin (m-1) \theta \\
\vdots \\
\sin \theta \\
0
\end{array}\right]
\end{aligned}
$$

and $D(\theta, r, \boldsymbol{b})=d\left[\arg b\left(r e^{j \theta}\right)\right] / d \theta$, then along $\mathcal{C}$ polynomial $b(z)$ can be expressed as

$$
b\left(r e^{j \theta}\right)=b_{r}(\theta, r)+j b_{i}(\theta, r)
$$

where

$$
\begin{aligned}
b_{r}(\theta, r) & =c_{r m}(\theta)+\boldsymbol{b}^{T} \boldsymbol{c}_{r}(\theta) \\
b_{i}(\theta, r) & =s_{r m}(\theta)+\boldsymbol{b}^{T} \boldsymbol{s}_{r}(\theta)
\end{aligned}
$$


and $D(\theta, r, \boldsymbol{b})$ is given by

$$
D(\theta, r, \boldsymbol{b})=\frac{b_{r}(\theta, r) t_{r}(\theta)+b_{i}(\theta, r) t_{i}(\theta)}{b_{r}^{2}(\theta, r)+b_{i}^{2}(\theta, r)}
$$

with $t_{r}(\theta)=m c_{r m}(\theta)+\boldsymbol{b}^{T} \boldsymbol{s}_{r}^{\prime}(\theta), t_{i}(\theta)=m s_{r m}(\theta)-$ $\boldsymbol{b}^{T} \boldsymbol{c}_{r}^{\prime}(\theta)$.

Notice that $D(\theta, r, \boldsymbol{b})$ is an even function of $\theta$. This in conjunction with (2)-(4) imply that $b(z)$ is $r$-Schur if and only if

$$
\frac{1}{\pi} \int_{0}^{\pi} D(\theta, r, \boldsymbol{b}) d \theta=m
$$

Two remarks are now in order. First, for a given radius $r \leq 1$, the stability condition in (5) is a single equality constraint that does not depend on parameter $\theta$ (because of the integration there) and the constraint is explicit in terms of the polynomial coefficients. Second, if $b(z)$ is known and $r$-Schur, then perturbed polynomial $\hat{b}(z)+\beta(z)$ with $\beta(z)=$ $\beta_{1} z^{m-1}+\cdots+\beta_{m}$ remains $r$-Schur if and only if

$$
\frac{1}{\pi} \int_{0}^{\pi} D(\theta, r, \boldsymbol{b}+\boldsymbol{\beta}) d \theta=m
$$

where $\boldsymbol{\beta}=\left[\begin{array}{lll}\beta_{1} & \ldots & \beta_{m}\end{array}\right]^{T}$. With $\boldsymbol{b}$ and $r$ fixed, (6) is an explicit sufficient and necessary condition for $\boldsymbol{\beta}$ to ensure the $r$-Schurness of $b(z)+\beta(z)$.

If we define function $N(\boldsymbol{b}, r)$ as

$$
N(\boldsymbol{b}, r)=\frac{1}{\pi} \int_{0}^{\pi} D(\theta, r, \boldsymbol{b}) d \theta
$$

then the analysis above shows that $N(\boldsymbol{b}, r)$ is an integervalued piecewise-constant function in the entire parameter space where $\boldsymbol{b}$ resides. The stability condition in (6) can now be stated as $N(\boldsymbol{b}+\boldsymbol{\beta}, r)=m$ for a stable perturbation $\boldsymbol{\beta}$. For illustration purposes, we follow [5] to consider $b(z)=$ $z^{2}-0.5 z+0.6$ and let $\boldsymbol{b}=\left[\begin{array}{ll}-0.5 & 6\end{array}\right]^{T}, \boldsymbol{\beta}=\left[\begin{array}{ll}\beta_{1} & \beta_{2}\end{array}\right]^{T}$. It can be verified that $b(z)$ is stable and $\tilde{b}(z)=b(z)+$ $\beta(z)=z^{2}+\left(\beta_{1}-0.5\right) z+\left(\beta_{2}+0.6\right)$ is stable if and only if $\beta_{2}<0.4, \beta_{1}-\beta_{2}<2.1$, and $\beta_{1}+\beta_{2}>-1.1$. The stability region in this case is a triangle shown in Fig. 1. Function $N(\boldsymbol{b}+\boldsymbol{\beta}, r)$ is then evaluated in the rectangular region $\left\{-1.5 \leq \beta_{1} \leq 2.5,-1.6 \leq \beta_{2} \leq 0.4\right\}$, and the evaluation results are depicted in Fig. 2.

It is observed that the region where $N(\boldsymbol{b}+\boldsymbol{\beta}, 1)$ assumes the value of 2 , i.e., the region where the corresponding polynomial is 1-Schur, is identical to the triangle in Fig. 1. The stability regions for this particular $b(z)$ obtained based on Rouchés theorem and positive realness are proper sub-regions inside the triangle and can be found in [5].

\section{Weighted Least-SQuares Design}

Consider the transfer function of an IIR digital filter

$$
H(z)=\frac{a(z)}{z^{n-m} b(z)}=\frac{\sum_{i=0}^{n} a_{i} z^{n-i}}{z^{n-m} \sum_{i=0}^{m} b_{i} z^{m-i}}
$$

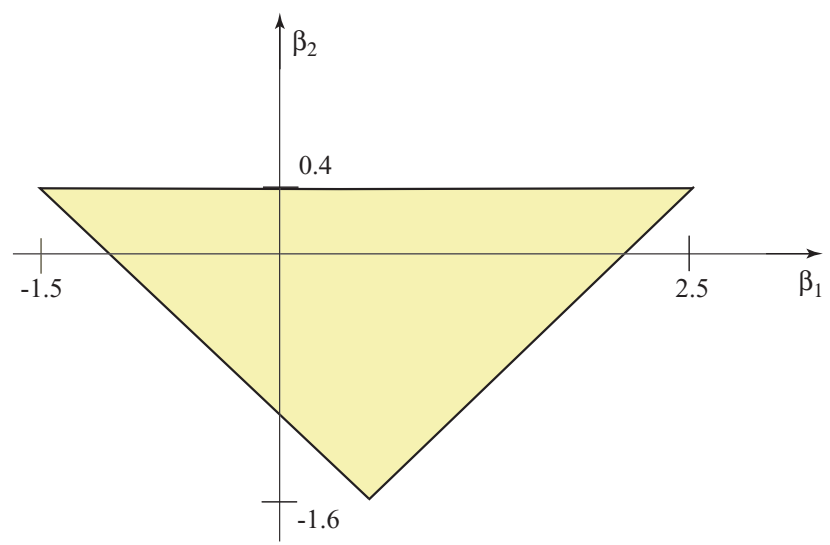

Fig. 1. Stability region for $\tilde{b}(z)$.

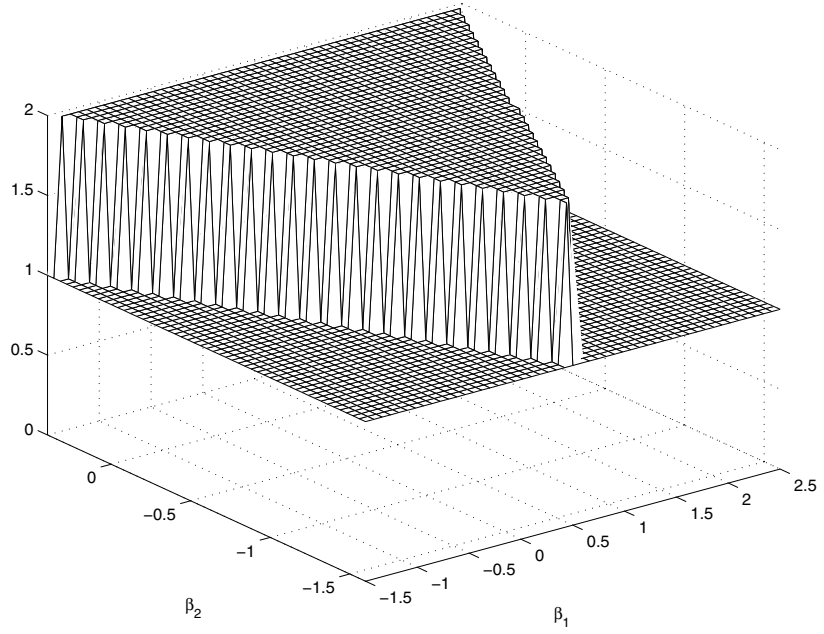

Fig. 2. Function $N(\boldsymbol{b}+\boldsymbol{\beta}, 1)$.

where $0 \leq m \leq n$. The filter is assigned $(n-m)$ poles at the origin as it was found beneficial for the design of several types of digital filters [3]. The frequency response of the filter can be expressed as $H(\omega, \boldsymbol{x})=H_{r}(\omega, \boldsymbol{x})+j H_{i}(\omega, \boldsymbol{x})$ with

$$
\begin{aligned}
H_{r}(\omega, \boldsymbol{x}) & =\frac{\boldsymbol{a}^{T} \hat{\boldsymbol{c}}(\omega) \boldsymbol{b}_{r}(\omega, 1)+\boldsymbol{a}^{T} \hat{\boldsymbol{s}}(\omega) \boldsymbol{b}_{i}(\omega, 1)}{b_{r}^{2}(\omega, 1)+b_{i}^{2}(\omega, 1)} \\
H_{i}(\omega, \boldsymbol{x}) & =\frac{\boldsymbol{a}^{T} \hat{\boldsymbol{s}}(\omega) \boldsymbol{b}_{r}(\omega, 1)-\boldsymbol{a}^{T} \hat{\boldsymbol{c}}(\omega) \boldsymbol{b}_{i}(\omega, 1)}{b_{r}^{2}(\omega, 1)+b_{i}^{2}(\omega, 1)} \\
\boldsymbol{a} & =\left[\begin{array}{c}
a_{0} \\
\vdots \\
a_{n}
\end{array}\right], \boldsymbol{x}=\left[\begin{array}{c}
\boldsymbol{a} \\
\boldsymbol{b}
\end{array}\right], \\
\hat{\boldsymbol{c}}(\omega) & =\left[\begin{array}{c}
\cos n \omega \\
\vdots \\
\cos \omega \\
1
\end{array}\right], \hat{\boldsymbol{s}}(\omega)=\left[\begin{array}{c}
\sin n \omega \\
\vdots \\
\sin \omega \\
0
\end{array}\right]
\end{aligned}
$$

and $\boldsymbol{b}, b_{r}(\omega, 1)$, and $b_{i}(\omega, 1)$ are defined in (4).

In a weighted least squares design, one seeks an $H(z)$ in 
(7) that solves the constrained problem

$$
\begin{array}{cl}
\underset{\boldsymbol{x}}{\operatorname{minimize}} & \int_{-\pi}^{\pi} W(\omega)\left|H(\omega, \boldsymbol{x})-H_{d}(\omega)\right|^{2} d \omega \\
\text { subject to: } & b(z) \text { is } r \text {-Schur }
\end{array}
$$

where $H_{d}(\omega)=H_{d r}(\omega)+j H_{d i}(\omega)$ denotes a desired frequency response and $W(\omega) \geq 0$ is a known weighting function. In the $k$ th iteration of an iterative solution process,

$H\left(\omega, \boldsymbol{x}_{k}+\boldsymbol{\delta}\right) \approx H\left(\omega, \boldsymbol{x}_{k}\right)+\boldsymbol{g}_{k}^{T}(\omega) \boldsymbol{\delta}$

where

$$
\begin{aligned}
\boldsymbol{g}_{k}(\omega) & =\nabla H\left(\omega, \boldsymbol{x}_{k}\right)=\nabla H_{r}\left(\omega, \boldsymbol{x}_{k}\right)+j \nabla H_{i}\left(\omega, \boldsymbol{x}_{k}\right) \\
& =\boldsymbol{g}_{k r}(\omega)+j \boldsymbol{g}_{k i}(\omega)
\end{aligned}
$$

Hence

$$
W(\omega)\left|H\left(\omega, \boldsymbol{x}_{k}+\boldsymbol{\delta}\right)-H_{d}(\omega)\right|^{2} \approx\left\|\boldsymbol{G}_{k}(\omega) \boldsymbol{\delta}+\boldsymbol{e}_{k}(\omega)\right\|^{2}
$$

where

$$
\begin{aligned}
\boldsymbol{G}_{k}(\omega)= & W^{1 / 2}(\omega)\left[\begin{array}{c}
\boldsymbol{g}_{k r}^{T}(\omega \\
\boldsymbol{g}_{k i}^{T}(\omega)
\end{array}\right] \\
\boldsymbol{e}_{k}(\omega)= & W^{1 / 2}(\omega)\left[\begin{array}{c}
e_{k r}(\omega) \\
e_{k i}(\omega)
\end{array}\right] \\
e_{k r}(\omega)= & H_{r}\left(\omega, \boldsymbol{x}_{k}\right)-H_{d r}(\omega) \\
e_{k i}(\omega)= & H_{i}\left(\omega, \boldsymbol{x}_{k}\right)-H_{d i}(\omega) \\
\boldsymbol{\delta}= & {\left[\begin{array}{c}
\alpha_{0} \\
\vdots \\
\alpha_{n} \\
\beta_{1} \\
\vdots \\
\beta_{m}
\end{array}\right]=\left[\begin{array}{c}
\boldsymbol{\alpha} \\
\boldsymbol{\beta}
\end{array}\right] }
\end{aligned}
$$

In this way, the problem in (9) is reduced to

$$
\begin{array}{cl}
\underset{\boldsymbol{\delta}}{\operatorname{minimize}} & \int_{-\pi}^{\pi}\left\|\boldsymbol{G}_{k}(\omega) \boldsymbol{\delta}+\boldsymbol{e}_{k}(\omega)\right\|_{2}^{2} d \omega \\
\text { subject to: } & \|\boldsymbol{\alpha}\|,\|\boldsymbol{\beta}\| \text { small } \\
& b(z) \text { is } r \text {-Schur }
\end{array}
$$

whose solution, $\boldsymbol{\delta}_{k}$, is in turn used to update $\boldsymbol{x}_{k}$ to $\boldsymbol{x}_{k+1}=$ $\boldsymbol{x}_{k}+\boldsymbol{\delta}_{k}$. This process continues until $\left\|\boldsymbol{\delta}_{k}\right\|_{2}$ becomes smaller than a prescribed tolerance $\varepsilon$. Notice the constraints on the magnitude of $\boldsymbol{\alpha}, \boldsymbol{\beta}$ as imposed in (11b) - they are needed to validate the linear approximation of $H\left(\omega, \boldsymbol{x}_{k}+\boldsymbol{\delta}\right)$. The norm in (11b) can be either 2-norm or infinity-norm.

A natural way to deal with (11c) that is consistent with linearly approximating $H\left(\omega, \boldsymbol{x}_{k}+\boldsymbol{\delta}\right)$ is to consider the linear approximation of the stability condition in (6), i.e.,

$$
\begin{aligned}
& \frac{1}{\pi} \int_{0}^{\pi} D\left(\theta, r, \boldsymbol{b}_{k}+\boldsymbol{\beta}\right) d \theta \\
& \approx \frac{1}{\pi} \int_{0}^{\pi} D\left(\theta, r, \boldsymbol{b}_{k}\right) d \theta+\boldsymbol{\beta}^{T} \frac{1}{\pi} \int_{0}^{\pi} \nabla D\left(\theta, r, \boldsymbol{b}_{k}\right) d \theta
\end{aligned}
$$

which in conjunction with (5) leads to $\boldsymbol{v}^{T}\left(r, \boldsymbol{b}_{k}\right) \boldsymbol{\beta}=0$ with

$$
\boldsymbol{v}\left(r, \boldsymbol{b}_{k}\right)=\frac{1}{\pi} \int_{0}^{\pi} \nabla D\left(\theta, r, \boldsymbol{b}_{k}\right) d \theta
$$

By employing the infinity-norm in (11b), problem (11) now becomes

$$
\begin{aligned}
\operatorname{minimize} & \boldsymbol{\delta}^{T} \boldsymbol{P}_{k} \boldsymbol{\delta}+2 \boldsymbol{\delta}^{T} \boldsymbol{p}_{k} \\
\text { subject to: } & \left|\alpha_{i}\right| \leq q_{a} \quad i=0, \ldots, n \\
& \left|\beta_{i}\right| \leq q_{b} \quad i=1, \ldots, m \\
& \boldsymbol{v}^{T}\left(r, \boldsymbol{b}_{k}\right) \boldsymbol{\beta}=0
\end{aligned}
$$

where

$$
\begin{gathered}
\boldsymbol{P}_{k}=\int_{-\pi}^{\pi} \boldsymbol{G}_{k}^{T}(\omega) \boldsymbol{G}_{k}(\omega) d \omega \\
\boldsymbol{p}_{k}=\int_{-\pi}^{\pi} \boldsymbol{G}_{k}^{T}(\omega) \boldsymbol{e}_{k}(\omega) d \omega
\end{gathered}
$$

which is obviously a convex quadratic programming (QP) problem.

\section{Weighted Minimax Design}

The design problem in this case can be described as

$$
\underset{\boldsymbol{x}}{\operatorname{minimize}} \underset{-\pi \leq \omega \leq \pi}{\operatorname{maximize}} W(\omega)\left|H(\omega, \boldsymbol{x})-H_{d}(\omega)\right|
$$

Here an iterative design strategy similar to that in Sec. III can be adopted to yield the following optimization problem for vector $\boldsymbol{\delta}_{k}$ that updates the $k$ th intermediate design $\boldsymbol{x}_{k}$ to $\boldsymbol{x}_{k+1}=\boldsymbol{x}_{k}+\boldsymbol{\delta}_{k}$ :

$$
\begin{aligned}
\operatorname{minimize} & \eta \\
\text { subject to: } & \left\|\boldsymbol{G}_{k}\left(\omega_{i}\right) \boldsymbol{\delta}+\boldsymbol{e}_{k}\left(\omega_{i}\right)\right\|_{2} \leq \eta \\
& \text { for } i=1, \ldots, K \\
& \|\boldsymbol{\alpha}\|_{2} \leq q_{a},\|\boldsymbol{\beta}\|_{2} \leq q_{b} \\
& \boldsymbol{v}^{T}\left(r, \boldsymbol{b}_{k}\right) \boldsymbol{\beta}=0
\end{aligned}
$$

where a dense grid points $\left\{\omega_{i}, i=1, \ldots, K\right\} \subset[-\pi, \pi]$ are used in (14b) and 2-norm is used to measure the smallness of vectors $\boldsymbol{\alpha}$ and $\boldsymbol{\beta}$ in (14c). If we treat the upper bound $\eta$ as an auxiliary variable (in addition to $\delta$ ), then (14) is a second-order cone programming (SOCP) problem [9]. Notice that unlike the QP problem in (12) where the total number of (linear) constraints is $2(n+m)+3$, the number of constraints involved in (14) is usually much greater as $K>>(n+m)$.

\section{Design Examples}

As the first example, the weighted LS algorithm proposed in Sec. III was applied to design a lowpass IIR filter with $n=15, m=4$, normalized passband edge $f_{p}=0.4$, and normalized stopband edge $f_{a}=0.56$. The desired passband group delay was set to $\tau=12$. The pole radius was set to $r=0.84$, the bounds in (12b) and (12c) were set to $q_{a}=0.4$, $q_{b}=0.35$, and the weighting function

$$
W(\omega)= \begin{cases}1 & \text { for } \omega \in\left[0, f_{p}\right] \\ 2.6 & \text { for } \omega \in\left[f_{a}, 1\right]\end{cases}
$$

was used. The algorithm was implemented using MATLAB where the key command for solving problem (12) is quadprog. With $\varepsilon=10^{-6}$, it took the algorithm 15 iterations 
and 0.5 seconds CPU time on a Pentium $43.3 \mathrm{GHz} \mathrm{PC}$ to converge to the solution. The performance of the IIR filter obtained was evaluated in terms of the $L_{2}$ error in passband $e_{2 p}=0.8187 \times 10^{-5}, L_{2}$ error in stopband $e_{2 a}=0.1420 \times$ $10^{-5}$, average relative deviation in passband group delay $e_{g d}=0.0028$ and maximum modulus of the poles $=0.8309$. The filter's performance was compared with a counterpart IIR filter reported in [3] with the same $n, m, f_{p}, f_{a}$, and $\tau$ as above. The counterpart filter of [3] was evaluated in terms of $e_{2 p}=0.8720 \times 10^{-5}, e_{2 a}=0.1483 \times 10^{-5}, e_{g d}=0.0028$ and maximum modulus of the poles $=0.8263$. The amplitude responses and passband group delays of these two filters are depicted in Fig. 3 with solid line representing the filter obtained by the proposed method and dashed line for the filter of [3].
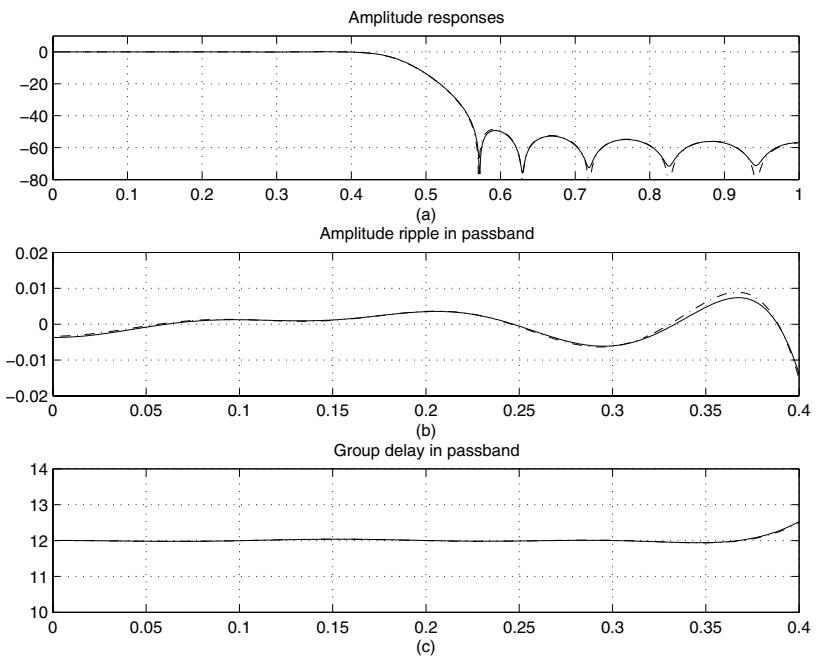

Fig. 3. Amplitude responses, passband ripples and passband group delays for the first example.

In the second example, the weighted minimax algorithm described in Sec. IV was applied to design a lowpass IIR filter with $n=m=12, f_{p}=0.5, f_{a}=0.6$, and $\tau=15.9$. The pole radius was set to $r=0.94$, the bounds in (14c) were set to $q_{a}=1.62$ and $q_{b}=1.55$, and $W(\omega) \equiv 1$ for $\omega \in\left[0, f_{p}\right]$ and $\left[f_{a}, 1\right]$. The algorithm was implemented using MATLAB toolbox SeDuMi version 1.1R2 [11]. With $\varepsilon=2 \times 10^{-5}$ and $K=200$, it took the algorithm 16 iterations and 4.77 seconds CPU time to converge to the solution whose performance was evaluated in terms of maximum passband ripple $e_{r p}=0.0137$, minimum stopband attenuation $e_{r a}=37.2051 \mathrm{~dB}$, average relative deviation in passband group delay $e_{g d}=0.0088$, and maximum modulus of the poles $=0.9327$. The performance of the well-known Deczky filter [10] with the same $n, m, f_{p}, f_{a}$, and $\tau$ was used as the benchmark for comparison purposes. The Deczky filter offers $e_{r p}=0.0549, e_{r a}=31.7603, e_{g d}=$ 0.0233 , and maximum modulus of the poles $=0.8929$. The amplitude responses and passband group delays of these two filters are given in Fig. 4 with solid lines for the IIR filter obtained by the present method and dashed lines for the Deczky filter.
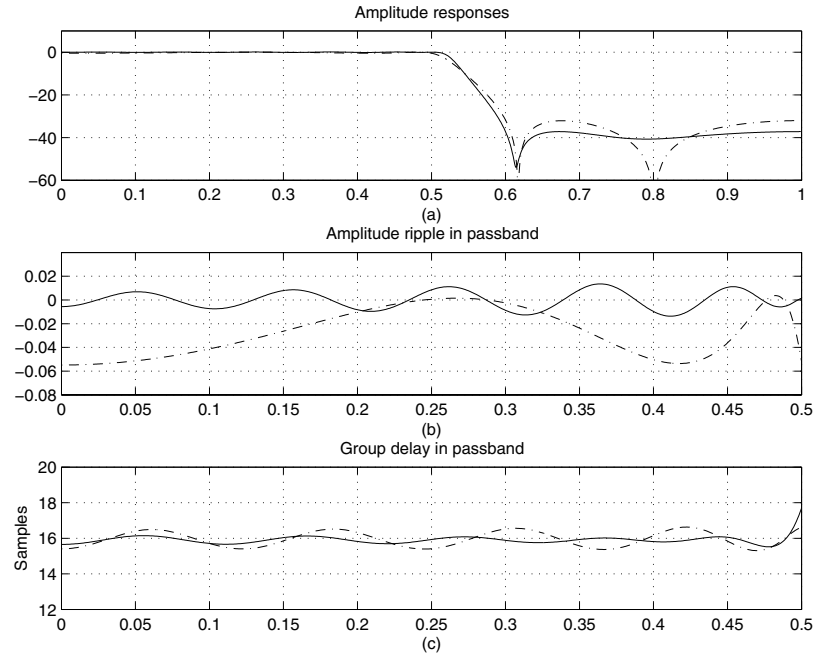

Fig. 4. Amplitude responses, passband ripples, and passband group delays for the second example.

\section{ACKNOWLEDGEMENT}

The author is grateful to the Natural Sciences and Engineering Research Council of Canada for supporting this work.

\section{REFERENCES}

[1] A. T. Chottera and G. A. Jullien, "A linear programming approach to recursive digital filter design with linear phase," IEEE Trans. Circuits Syst., vol. 29, pp. 139-149, Mar. 1982.

[2] W.-S. Lu, S.-C. Pei, and C.-C. Tseng, "A weighted least squares method for the design of stable 1-D and 2-D IIR filters," IEEE Trans. Signal Processing, vol. 46, pp. 1-10, Jan. 1998.

[3] M. C. Lang, "Least-squares design of IIR filters with prescribed magnitude and phase response and a pole radius constraint," IEEE Trans. Signal Processing, vol. 48, pp. 3109-3121, Nov. 2000.

[4] W.-S. Lu, "Design of stable minimax IIR digital filters using semidefinite programming," Proc. ISCAS, vol. 1, pp. 355-358, May 2000.

[5] B. Dumitrescu, "On convex stability domain and optimization of IIR filters," Proc. EUSIPCO, vol. 2, pp. 191-194, Sept. 2002.

[6] W.-S. Lu and T. Hinamoto, "Optimal design of IIR digital filters with robust stability using conic-quadratic-programming updates," IEEE Trans. Signal Processing, vol. 51, pp. 1581-1592, June 2003.

[7] B. Dumitrescu, "Least squares optimization of 2-D IIR filters," Proc. 6th Nordic Signal Processing Symp., pp. 33-36, Espoo, Finland, June 2004.

[8] H. Silverman, Complex Variables, Houghton Mifflin Co., 1975.

[9] M. S. Lobo, L. Vandenberghe, S. Boyd, and H. Lebret, "Applications of second-order cone programming," Linear Algebra and Its Applications, vol. 248, pp. 193-228, Nov. 1998.

[10] A. G. Deczky, "Synthesis of recursive digital filters using the minimum p-error criterion," IEEE Trans. Audio Electroacoust., vol. 20, pp. $257-$ 263, Oct. 1977.

[11] J. F. Sturm, "Using SeDuMi 1.02, a MATLAB toolbox for optimization over symmetric cones," Optimization Methods and Software, vol. 1112 , pp. 625-653, 1999. (version 1.1R2 of SeDuMi is available at http://sedumi.mcmaster.ca/) 